\title{
AVM's and Aneurysms, Hybrid Brain Vascular Surgery: A Case Report
}

Arturo Muñoz Cobos*, Aldo F Hernandez Valencia, Guadalupe Guerrero Avendaño, Luis Graniel Palafox, Rocio Enriquez Garcia, Jose Luis Navarro Olverea and Francisco Velasco Campos

Vascular Neurosurgeon, Clinic Brain Vascular Surgery, National Autonomous University of Mexico, México

\begin{abstract}
This review tries to describe in a didactical and practical manner the advantages of a combined approach, showing in two cases that Endovascular Therapy and Microsurgical Brain Surgery, can work together, as the best procedure, trying to save resources, time and complications that occur when both strategies work separately. There are reports in different countries about the combination of therapies. Several years ago, we treated many patients working in a conventional surgical room, with some limitations, but recently we have been working in a hybrid surgical room, making the diagnosis, surgical and endovascular treatment in one step. As we know in vascular diseases, saving time is mandatory, and we have improved our own outcomes in ruptured brain aneurysms, and when embolization and surgical resection has been needed for arteriovenous malformations (AVMs).
\end{abstract}

Keywords: Hybrid brain vascular surgery; Arteriovenous malformations; Aneurysms surgery; Embolization and surgery for AVMs and aneurysms

\section{Introduction}

Brain vascular diseases, like aneurysms and arteriovenous malformations, should be treated in the best surgical room, with the best resources and opportunely. Aneurysms and AVM's surgeries are a challenge, and the neurosurgeon needs to develop specific skills and knowledge to maximize effectiveness and safety of the patient.

When we talk about an aneurysm, we think in fine dissection and is mandatory to expose proximal and distal aneurysm neck and the parent artery. Sometimes in middle cerebral artery aneurysms, many surgeons feel comfortable because the proximal closure and complete trapping can be reached easily. Particularly in paraclinoid aneurysm trapping can be complicated, even when proximal closure of internal carotid artery, can be done in cervical segment, petrous or clinoid segments; as we know paraclinoid aneurysms have flow coming from internal carotid artery, ophthalmic artery, posterior communicating artery, anterior cerebral artery, and cavernous branches, doing trapping really complicated. In large and giant aneurysms, sometimes a surgeon needs clipping by remodeling with diverse types of clips, and can be difficult to identify the carotid wall from the aneurysm wall, beside atheroma and clots can dislodge and generate embolism, with ischemic complications, for these reasons, balloon-assisted clipping is the optimal option for a safer surgery [1-3].

Arteriovenous malformation is the most complex brain vascular disease needs comprehension of several anatomic details and hemodynamic behavior. In various places in high flow AVM or some AVM Spetzler-Martin grade III, frequently we need preoperative embolization for better treatment. In a classic way, people make preoperative embolization in hemodynamic room, usually days before surgery, causing sometimes brain edema by extravasation of embolization agent. In our institution, we make an MRI and preoperative DSA for planning the strategy, after that the following days we take the patient to the hybrid surgical room, and as the initial part of the treatment, embolization proceeds, basically to reduce flow into the arteriovenous malformation, which maneuver improves the surgical stage [4].

\section{Patients and Materials}

In these cases, from General Hospital Of Mexico, we describe the combination of endovascular therapy and microsurgical clipping in an unruptured ventral paraclinoid aneurysm describing the use of balloon occlusion technique, and we also describe a ruptured ventral temporal lobe AVM treated by embolization and resection in one step, pointing out the technique and advantages of "one-stopshop treatment". An Envoy 6 Fr treatment catheter is placed, under continuous irrigation with a hemostatic key in "Y" with infusion pump with solution for injection with dilution of 2000 IU regular heparin for every $1000 \mathrm{cc}$ of solution. For the case of AVM, we performed in coaxial microcatheterization process of afferents vessels, greater than the malformation nest and strict intranidal position, in blind spot under the surgical microscope, performing a preoperative embolization with either ONIX 18 (EV3 Covidien) and/or NBCA, proceeding immediately with the micro-surgical resection in the same surgical act. In the case of intracranial aneurysm we ascended with a compliant occlusion balloon Hiper Glad and/or Hiper Form $3.5 \times 15$ (EV3) until the occlusion of the aneurysm in its entirety and simultaneously under Road Mapping vision we proceed with the microsurgical clipping in real time. After the safe clipping we deflated the balloon and removed it later. The balloon serving as scaffolding for the parent vessel avoid the sacrifice of arterial diameter, which is always important in clipping under technique microsurgery [5]. For both procedures we used, the microscope Pentero 900, (Carl Zeiss Germany), and radio-translucent carbon fiber cranial support (Macquet).

\section{Aneurysm Case Report}

A 65 year old woman, with a familiar history of aneurysms, hypertension, obesity, and former smoking, presented with a symptom of a two-years atypical headache. The computed tomography did not

*Corresponding author: Arturo Muñoz Cobos, Vascular Neurosurgeon, Clinic Brain Vascular Surgery, National Autonomous University of Mexico, México, Tel: +5215585358665; E-mail: artmcobos@gmail.com

Received September 20, 2017; Accepted October 06, 2017; Published October 17, 2017

Citation: Cobos AM, Valencia AFH, Avendaño GG, Palafox LG, Garcia RE, et al (2017) AVM's and Aneurysms, Hybrid Brain Vascular Surgery: A Case Report. J Vasc Med Surg 5: 341. doi: 10.4172/2329-6925.1000341

Copyright: (c) 2017 Cobos AM, et al. This is an open-access article distributed under the terms of the Creative Commons Attribution License, which permits unrestricted use, distribution, and reproduction in any medium, provided the original author and source are credited. 
Citation: Cobos AM, Valencia AFH, Avendaño GG, Palafox LG, Garcia RE, et al. (2017) AVM's and Aneurysms, Hybrid Brain Vascular Surgery: A Case Report. J Vasc Med Surg 5: 341. doi: 10.4172/2329-6925.1000341

Page 2 of 4

show any abnormality and the DSA showed a right ventral paraclinoid aneurysm (Figure 1). The patient was admitted to our hospital, she received all information about the procedure, we explain everything and she accepted the proposed treatment.

\section{Surgical Procedure}

The patient was taken to the Hybrid surgical room, and general anesthesia was induced by intravenous administration of sufentanil $(0.4 \mathrm{mcg} / \mathrm{kg})$, propofol $(2 \mathrm{mg} / \mathrm{kg})$, rocuronium $(0.6 \mathrm{mg} / \mathrm{kg})$. The head was fixed with three pin radio-translucent carbon fiber cranial support, without extension $0^{\circ}$ and rotation $5^{\circ}$. A right pterional approach was performed, complete dissection of Sylvian cistern with exposure of the aneurysm was achieved, with identification of carotid artery bifurcation, posterior communicating artery, superior hypophyseal artery (Figure 2 ). Resection of the anterior clinoid process was not needed. The craniotomy was covered to proceed with the endovascular procedure. Thorough a right femoral puncture and fluoroscope navigation the balloon catheter and their landmarks were positioned at the level of the aneurysm neck, and inflated to corroborate the correct position (Figure 3 ). In order to diminish the embolism risk, a saline solution $0.9 \%$, containing heparin dosage of 5,000 U/L was infused continually [6,7].

The microscope was positioned again into the surgical field for microsurgical clipping, then the balloon was inflated. After corroboration that trapping was achieved, the aneurysm was clipped with a fenestrated clip, without complication, then the balloon
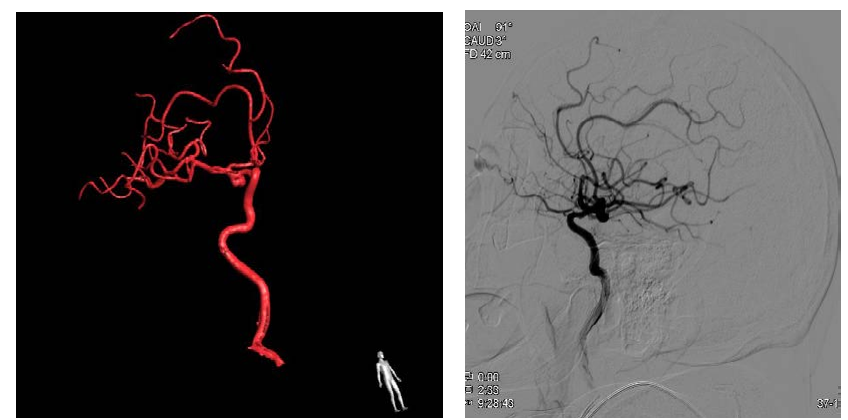

Figure 1: Digital substraction angiography in 3D reconstruction and latera projection showing a right ventral paraclinoid aneurysm.

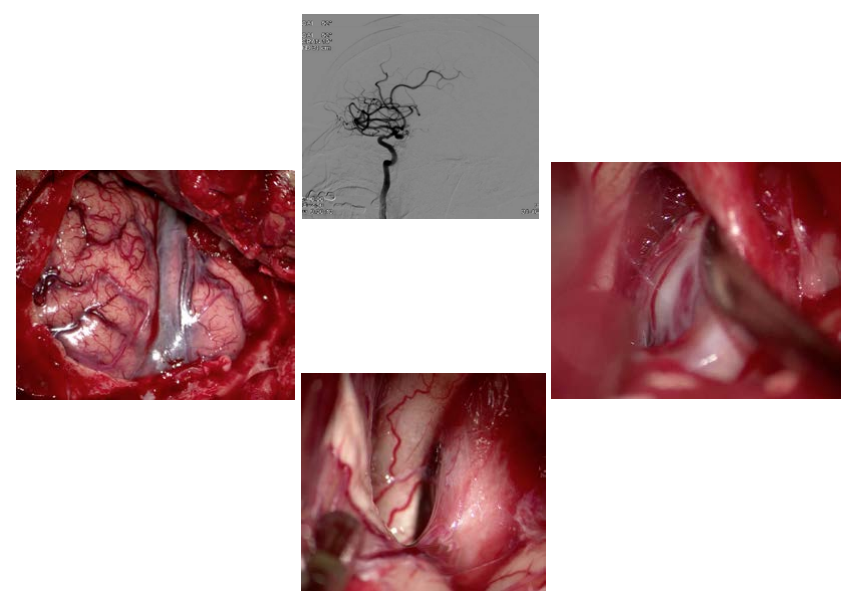

Figure 2: Rigth pterional approach was performed showing the Silvian veins, the complete disection of Silvian Cistern, where we can see the right optic nerve and supraclinoid internal carotid artery with the aneurysm in ventral position related to the superior hypophyseal artery. was deflated (Figure 4). After the clipping, we performed a DSA to corroborate the complete aneurysm exclusion, without compromise of parent vessels (Figure 4). The closure was performed in a conventional manner. The patient spent 24 hours in Intensive Care Unit, five more days in hospitalization and then she was discharged. In first month the patient had headache, and it was treated with painkillers, without any other symptom. Four months later a Rankin score was 0 .

\section{Arteriovenous Malformation Case report}

A 70 years old man was admitted to our hospital, with a history of sudden headache, nausea and vomiting, mild drowsiness, dysphasia, and right hemiparesis. Computed Tomography shows a temporal hematoma, and MRI revealed the AVM in the ventral surface of the left temporal lobe (Figure 5). Digital Subtraction Angiography (DSA) revealed an Arteriovenous Malformation with a compact nidus, and multiple feeder arteries from posterior cerebral artery, middle cerebral artery, and external carotid artery through middle meningeal artery (Figure 6). After planning the best treatment modality, we talk to the patient, about risks with and without surgery, and he accepted our procedure [8]

As previous description in aneurysm surgery the patient was taken to the Hybrid surgical room, and general anesthesia was induced by intravenous administration of sufentanil $(0.4 \mathrm{mcg} / \mathrm{kg})$, propofol $(2$ $\mathrm{mg} / \mathrm{kg})$, rocuronium $(0.6 \mathrm{mg} / \mathrm{kg})$. Thorough a right femoral puncture and fluoroscope navigation a microcatheter was introduced into the
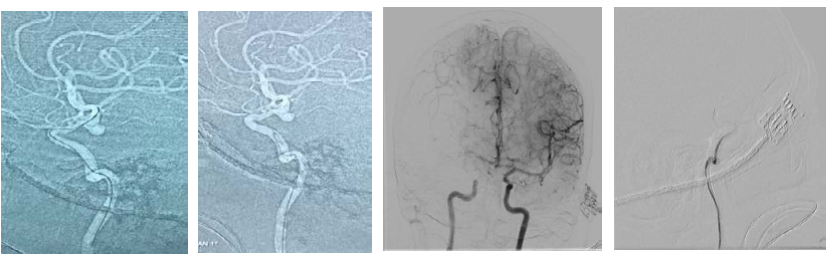

Figure 3: The lateral projection of DSA shows the balloon landmarks at the aneurysm neck, after inflating the balloon we can see the complete trapping in the anteroposterior and lateral DSA projections.
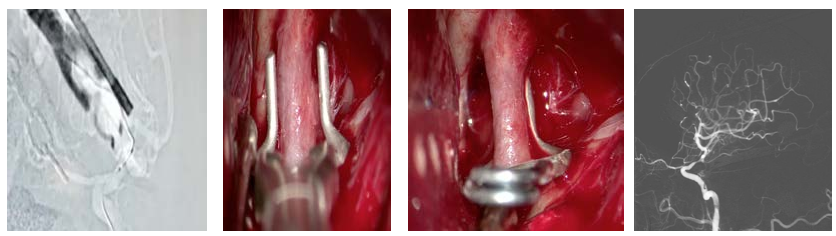

Figure 4: Under Road Mapping and microsurgical simultaneous vision the fenestrated clip was colocated, the balloon was deflated, and the lateral projection DSA of internal carotid artery shows the total exclusion of the aneurysm.
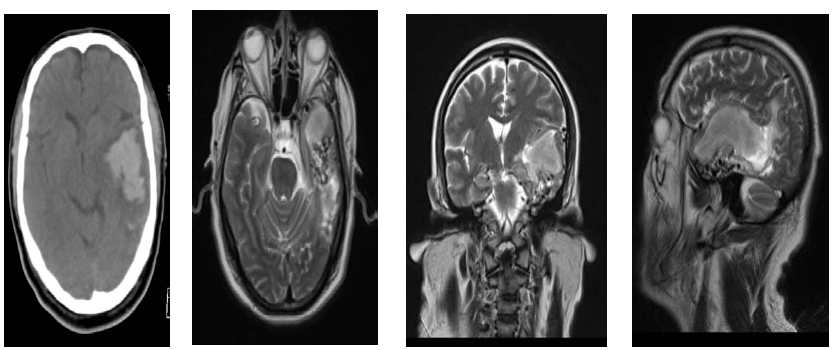

Figure 5: CT scan revealed a left temporal hematoma with mass effect. The axial, coronal and lateral T2 wighted MRI images show the hematoma and flow voids, corresponding to the left temporal AVM. 

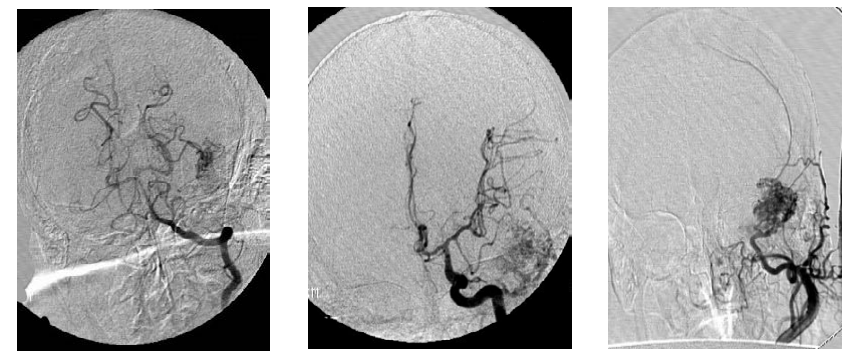

Figure 6: Anteroposterior angiogram of vertebrobasilar arteries, left internal carotid artery and left external carotid artery. Note the supply by three arterial systems.
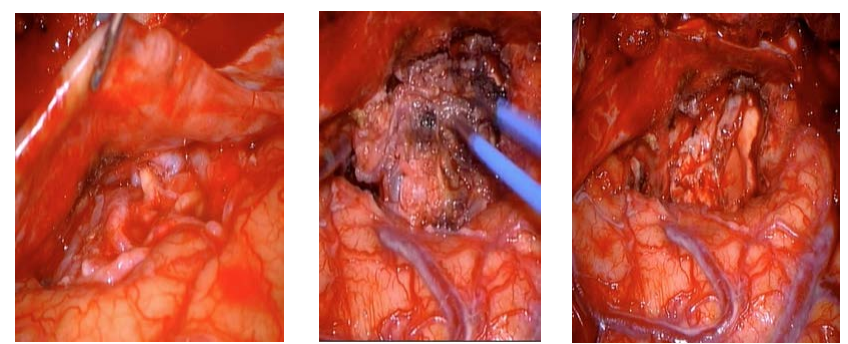

Figure 7: Microsurgical images of left temporal AVM, with extensive sulci disection, surrounding the AVM from brain tissue, allowing identify the feeding arteries for the complete resection.
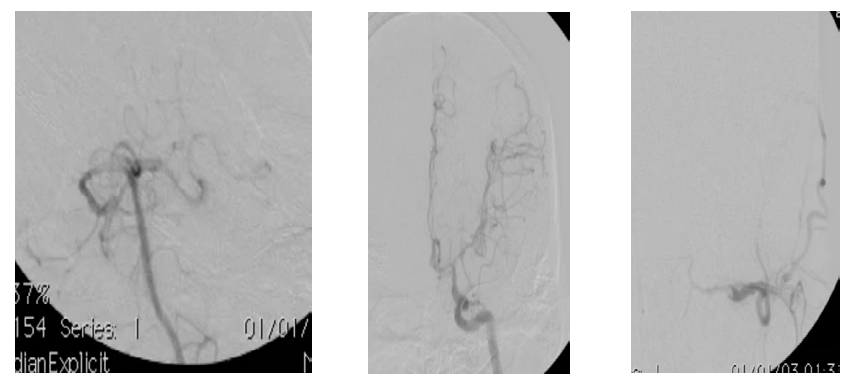

Figure 8: Posoperative angiogram from vertebrobasilar system, left internal carotid artery and left external carotid artery showing the complete AVM resection.

external carotid artery and middle meningeal artery, and supraselective embolization was performed with NBCA. The head was fixed with three pin radio-translucent carbon fiber cranial support. In this case the head was positioned with elevation $35^{\circ}$, rotation $30^{\circ}$ and lateral flexion, we chose a trans-zygomatic approach to expose the lateral and ventral surface of the left temporal lobe. We made a Dandy's incision, dissecting the temporal muscle and fascia in a conventional manner, we exposed the zygomatic arch and we performed an osteotomy improving the surgical view to the sub temporal exposure [7-9].

Arteriovenous malformation was exposed and resected by circumferential dissection, dividing feeding arteries and the hematoma was drained (Figure 7). At the end of the microsurgical procedure, before closure, we obtain a DSA showing a complete AVM resection (Figure 8).

\section{Discussion}

In different countries and hospitals, neurosurgeons try to offer Hybrid surgery, in two times, doing endovascular surgery in hemodynamic room, taking this patient to the surgical room for the microsurgical procedure. Nowadays we prefer the term "one stop shop treatment", which means that the patient has all modalities of treatment separated or in combination, in the same room, having different advantages. Before Hybrid surgical room, in our hospital it took two or three days to have a DSA, angio-CT and proceed with the surgery. Now our patients with subarachnoid hemorrhage arrive to our hospital and as soon as they are admitted and properly stabilized, we take them to the $\mathrm{x}$-ray department to obtain the angio-CT, if it shows an aneurysm, that is enough to take the patient to the hybrid surgical room, there we obtain a DSA, and clipping the aneurysm, and as soon as we set the clip, we obtain a DSA again to corroborate the aneurysm exclusion. We think when we have a tool that permit to have a control in the surgical room like Indocyanine green, digital subtraction angiography, Doppler ultrasound, the vessels manipulation is less, lowing the vasospasm risk. The digital subtraction angiography allows to see aneurysm exclusion, all brain arteries and vasospasm management. About vasospasm we treat all our patient with chemical or mechanical angioplasty in the same procedure, and in the same surgical room.

About brain arteriovenous malformations, we never operate them in acute phase, unless the patient is in bad clinical conditions. As we describe in aneurysms treating, we manage AVM's with a combination of techniques. Previously people have described the role of embolization in $\mathrm{AVM}^{\prime}$ 's treatment for:

- Reduce AVM flow for surgery,

- Reduce AVM volume for radiosurgery,

- Palliative treatment and

- Curative.

The clearest role of embolization for us is the first one, reducing flow for surgery. When we decided to treat a patient with an AVM, as protocol states, we obtain a DSA and MRI to plan the surgery. In parietal AVM's, Grade III, High flow, cerebellar AVM's we always use preoperative embolization, in the same surgical room, and when the microsurgical resection finished we obtain a DSA to corroborate the complete resection [5-8].

\section{Conclusion}

Brain vascular diseases need a multidisciplinary group, endovascular surgery and vascular microsurgery need to work together for treating both, aneurysms and AVM's. Nowadays in hybrid surgical rooms, patients have all treatment options separately or in combination. In brain aneurysm DSA in surgical room, allows diagnosis and treatment in one step, and we have the chance to make changes about sort of clip we want to apply. Brain AVM's embolization and surgery in one step, save time and resources, allowing us to see the complete AVM resection, and avoid brain edema and some other complications related with embolization days before surgery.

\section{References}

1. Thorell W, Rasmussen P, Perl J, Masaryk T, Mayberg M (2004) Balloonassisted microvascular clipping of paraclinoid aneurysms. Technical note. J Neurosurgery 100: 713-716.

2. Steiger HJ, Lins F, Mayer T, Schmid-Elsaesser R, Stummer W, et al. (2005) Temporary aneurysm orifice balloon occlusion as an alternative to retrograde suction decompression for giant paraclinoid internal carotid artery aneurysms: Technical note. Neurosurgery 56: ONS-E442.

3. Skrap M, Petralia B, Toniato G (2010) Temporary balloon occlusion during the surgical treatment of giant paraclinoid and vertebrobasilar aneurysms. Acta Neurochir 152: 435-442. 
Citation: Cobos AM, Valencia AFH, Avendaño GG, Palafox LG, Garcia RE, et al. (2017) AVM's and Aneurysms, Hybrid Brain Vascular Surgery: A Case Report. J Vasc Med Surg 5: 341. doi: 10.4172/2329-6925.1000341

Page 4 of 4

4. Elhammady MS, Nakaji P, Farhat H, Morcos JJ, Aziz-Sultan MA (2009) Ballon assisted clipping of a large paraclinoidal aneurysm; a salvage procedure. Neurosurgery 65: E1210-E1211.

5. Hu P, Zhang HQ, Li GL, He C, Bian LS, et al. (2015) A trapping-evacuation technique for giant carotid-ophthalmic segment aneurysm clipping in a hybrid operating theater. Journal of Clinical Neuroscience 22: 1184-1187.

6. Choudhri O, Mukerji N, Steinberg GK (2013) Combined endovascular and microsurgical management of complex cerebral aneurysms. Frontiers in Neurology 4: 108.
7. Fernandes ST, Alves RV, Doria-Netto HL, Junior PP, Rivau FR, et al. (2016) Treatmente of complex intracranial aneurysm: Case report of the simultaneous use of endovascular and microsurgical techniques. Surgical Neurology International.

8. Bradac GB (2011) Cerebral Angiography. Normal Anatomy and Patology, Springer.

9. Lawton M (2014) Seven AVMs Tenets and Techniques for Resection. Thieme 